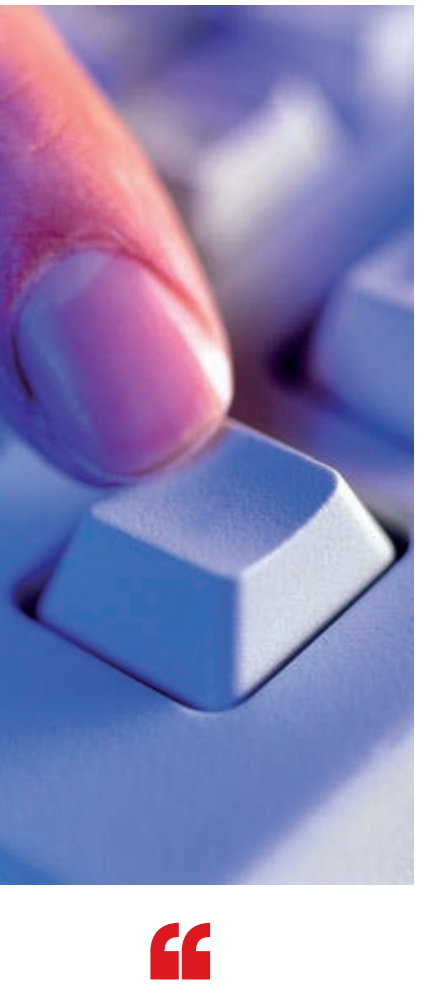

...tandem

repeat-containing

promoters have

the potential

to contribute

substantially to

the evolvability of

gene expression.

\title{
Hit repeat for evolvability
}

Along with changes that affect protein sequence, alterations in regulatory regions make important contributions to organismal evolution. Findings in yeast now suggest that variable tandem repeat (TR) sequences in promoter regions are a previously unrecognized cause of variability in transcription levels. This mechanism might make a substantial contribution to the evolvability of gene expression.

Although many TRs are intergenic, they can also reside in coding and regulatory regions. Vinces and colleagues found that as many as $25 \%$ of promoters in the sequenced Saccharomyces cerevisiae strain S288C contain one or more TR, and that many promoter TRs have variable numbers of repeat units between $S$. cerevisiae strains. They estimate that the frequency at which variation in TRs occurs is 40 times greater than that of insertion-deletion and point mutations in non-repetitive sequences.

Does this variation in TRs affect gene expression? The authors showed that orthologous genes with TR-containing promoters that are variable between related yeast species show greater divergence in transcriptional activity than genes with promoters that lack TRs. Furthermore, experiments using engineered gene constructs showed that increasing the number of repeat units in promoter
TRs increases expression up to a cut-off point, after which expression declines. For one gene, SDT1, which confers resistance to a nucleoside analogue, varying the number of repeat units in the promoter had functional consequences, with differences in TR length correlating with differences in the growth ability of cells.

The authors also provide strong evidence for a role of promoter TR variability in evolvability. Using strains in which one of two selectable markers was placed downstream of a TR-containing promoter, they saw changes in TR length after just a few rounds of selection for increased expression of the marker. For both markers, TRs with 13 repeat units were favoured - corresponding to the most highly expressed engineered constructs. When the same strains were grown without selection, far fewer changes in TR length were seen, and those that occurred showed a broader distribution of lengths. Moreover, replacing the TR by a similar, non-repetitive sequence eliminated the appearance of mutants with increased expression.

How does variability in TR length affect transcription? Of the 1,455 TR-containing promoters that the authors identified, 113 contain known binding sites for transcription factors. The authors highlight one case in which the number of binding sites for a transcription factor is altered by TR length, which may therefore have an impact on transcription levels. However, an alternative explanation is needed for TRs that do not contain transcription factor binding sites. Most promoter TRs lie 200 bp upstream of the start codon, corresponding to the nucleosome-free promoter region - an observation that prompted the authors to postulate that TRs influence nucleosome positioning. In support of this, deleting the TR of SDT1 led to increased binding of nucleosomes in this region and also affected the positioning of downstream nucleosomes.

As they occur so frequently and have such high mutation rates, TR-containing promoters have the potential to contribute substantially to the evolvability of gene expression. Vinces and colleagues suggest that this mode of evolution may also occur in higher eukaryotes and might be particularly applicable to genes that are involved in environmental responses.

Louisa Flintoft

ORIGINAL RESEARCH PAPER Vinces, M. D. et al. Unstable tandem repeats in promoters confer transcriptional evolvability. Science $\mathbf{3 2 4}$, 1213-1216 (2009)

FURTHER READING Wray, G. A. The evolutionary significance of cis-regulatory mutations. Nature Rev. Genet. 8, 206-216 (2007) 